\title{
Determination of four additives in cakes by large volume gradient ion chromatography
}

\author{
Ye $\mathrm{Liu}^{1}$, Pei Gu${ }^{1}$, and Yaling $\mathrm{Liu}^{2 *}$ \\ ${ }^{1}$ Xiangtan Institute Food and Drug Control, Xiangtan, 411100, \\ ${ }^{2}$ Chinese Academic for Environmental Planning, Beijing, 100000
}

\begin{abstract}
This paper aims to establish a detection method for simultaneous determining four additives in flour products: one simultaneously determines the acesulfame, sodium cyclamate, bromate, and calcium propionate through the selection of ion chromatography pump flow rate, injection volume, eluent concentration, optimization of sample pretreatment and the influence of interfering ions. When using the pump flow rate of $0.8 \mathrm{ml} / \mathrm{min}, 200 \mu \mathrm{L}$ large volume injection, $0-14 \mathrm{~min}, 14-25$ min eluent concentration $30 \mathrm{mmol} / \mathrm{L}$ gradient elution, 25-26 min eluent concentration $6 \mathrm{mmol} / \mathrm{L}$, flour product is pulverized after addition of water vortex ultrasonic extraction, the supernatant by $\mathrm{C} 18$ solid phase extraction (SPE) columns and silver filter, $0.22 \mu \mathrm{m}$ water phase porous membrane testing on the machine, the detection limits of the four kinds of targets are $0.16 \mu \mathrm{g} / \mathrm{ml}, 0.19 \mu \mathrm{g} / \mathrm{ml}, 0.24 \mu \mathrm{g} / \mathrm{ml}$, and $0.38 \mu \mathrm{g} / \mathrm{ml}$, respectively; The standard deviations of retention time and peak area are calcium propionate, respectively $0.13 \sim 0.42 \%, 2.14 \sim 4.25 \%$; bromate $0.20 \sim 0.67 \%$, $1.33 \sim 4.52 \%$; sodium cyclamate $0.14 \sim 0.53 \%, 3.57 \sim 5.62 \%$; acesulfame potassium $0.64 \sim 0.93 \%, 0.42 \sim 5.81 \%$. This method uses water as the sole extraction solvent and can detect four additives in flour products in a single test. And the pretreatment process is also very simple. When it is applied to other food tests, some compounds may cause interference, which can be assisted by other testing methods.
\end{abstract}

Keywords: Ion chromatography; large volume; Gradient elution; Acesulfame potassium; Sodium cyclamate; Bromate; Calcium propionate.

\section{Introduction}

In accordance with the product range and health certificate unit of the food production license, flour products include foods such as moon cakes, breads, cakes etc. The flour products use grain, oil, sugar, and eggs as the main ingredients, together with appropriate amounts of auxiliary additives, and are all processed through procedures such as preparation, molding, cooking, and packaging. [1]. According to the National Food Safety Supervision and Sampling Inspection Rules, the values of additives in pastries are limited. Referring to GB 2760-2014 "National Food Safety Standard Food Additives Use Standards" [2], the additives of acesulfame K, sodium cyclamate and calcium propionate in cakes shall not exceed $0.3 \mathrm{~g} / \mathrm{kg}, 1.6 \mathrm{~g} / \mathrm{kg}, 2.5 \mathrm{~g} / \mathrm{kg}$. The national standards of the People's

\footnotetext{
${ }^{*}$ Corresponding author: Liuyaling127@hotmail.com
} 
Republic of China stipulate the corresponding detection methods for respective additives[3]-[6]. When national security inspecting, detection of specified additives requires different complex and cumbersome pre-treatments, testing methods are also different, including liquid chromatography, liquid chromatography - Mass spectrometry / mass spectrometry, liquid chromatography and gas chromatography. Because the requirements for laboratory hardware and personnel are high, the detection of related additives has always been a research hotspot. For example, Wu Lirong [7], Liu Fang et al [8], Zhou Bin et al [9], used different detection methods, such as UPLC , High performance liquid chromatography - evaporative light scattering detection method, ultrahigh performance liquid chromatography tandem mass spectrometry method for the determination of various additives in foods.

\section{Materials and methods}

\subsection{Instruments and reagents}

ICS900 ion chromatograph (Thermo Fisher), configuration: Diane AS-DV autosampler; Diane EGC III KOH RFICTM eluent automatic generator; conductivity detector; Chromelon 7 chromatography workstation; Mettler ME204E electronic balance; vortex mixer; ultrasonic cleaning; high-speed centrifuge ( 4000r / min ); C18 solid phase extraction column; solid phase extraction Ag column; 0.22 [mu $\mathrm{m}$ aqueous phase porous membrane.

Cyclamate reference material: No. SB05-218-2008, produced by the Ministry of Agriculture Environmental Protection Research and Testing Institute; Acesulfame K: No. SB05-217-2008, produced by the Ministry of Agriculture Environmental Protection Research and Testing Institute; Standard substance of bromate solution in water: Code GBW (E) 100200, produced by Beijing Coast Hongmeng Standard Material Technology Co., Ltd.; chlorochloronitrate sulfate standard material in water: Code GBW (E) 081207, produced by Shanghai Institute of Metrology and Testing Technology; benzoic acid sorbic acid saccharin Sodium mixed standard material:

Chromatographic conditions: Chromatographic column IonPac AS19 (4 × 250mm), chromatographic protection column IonPac AG19 $(4 \times 50 \mathrm{~mm})$, Diane AERS $5004 \mathrm{~mm}$ ion suppressor, suppressor current: $\mathrm{mA}$.

\subsection{Sample pretreatment}

Accurately weigh $1 \mathrm{~g}$ (accurate to $0.001 \mathrm{~g}$ ) of the crushed flour product into a $50.0 \mathrm{~mL}$ centrifuge tube, add $20 \mathrm{~mL}$ of high-purity water, vortex to mix, and ultrasonically extract for $20 \mathrm{~min}$. After removing, add water to the required mark, shake it and let it stand. Place the centrifuge tube on the centrifuge at $3000 \mathrm{r} / \mathrm{min}$ for $20 \mathrm{~min}$. The supernatant passes through C18 solid phase extraction (SPE), solid phase extraction Ag column to remove interfering chloride ions, test on the machine after filtration by the $0.22 \mu \mathrm{m}$ water-phase microporous membrane. 


\section{Results and discussion}

\subsection{Pump flow rate}

The effects of flow rates of $0.6,0.8,1.0,1.2$ and $1.6 \mathrm{ml} / \mathrm{min}$ on the separation efficiency of the four additives were tested. As the pump flow rate increases, the resolution decreases and the peak time becomes shorter. Because the retention times of calcium propionate, bromate, and sodium cyclamate are relatively close, when the pump flow rate is greater than $0.8 \mathrm{ml} / \mathrm{min}$, the resolution of the above three substances cannot be satisfied. When the flow rate is $0.6 \mathrm{ml} / \mathrm{min}$, the peak time is longer. After overall consideration, the pump flow rate is chosen as $0.8 \mathrm{ml} / \mathrm{min}$.

\subsection{Injection volume}

When using a lower pump flow rate, the peak time of the target is far away from the negative peak of the water. Therefore, the injection volume can be increased to increase the sensitivity of the detection. The injection volumes of $10,20,50,100,200,500 \mu \mathrm{L}$ are chosen respectively to test the influence on the response value. As the injection volume increases, the response value of the target value gradually increases. Because the detection sample contains more interfering ions, selecting an injection volume of $200 \mu \mathrm{L}$ not only meets the higher sensitivity but also ensures the degree of separation between the various components.

\subsection{Eluent concentration}

The effects of eluent concentrations of $6,8,12,15,20,30,50 \mathrm{mmol} / \mathrm{L}$ on the separation effect were tested. As the eluent concentration increases, the peak time shortens. The target calcium propionate, bromate, and sodium cyclamate have similar peak time, and the peak time is faster. When the concentration of the eluent is higher than $8 \mathrm{mmol} / \mathrm{L}$, the three targets cannot be well separated. When the concentration is $6 \mathrm{mmol} / \mathrm{L}$, the separation effect is better. The retention time of Acesulfame is long, and the peak only emerges until 40min. At 14min when three kinds of target peaks emerge, acesulfame peak emerges much earlier when eluent concentration increases; higher pressure column is achieved at higher eluent concentration. Using eluent concentration of $30 \mathrm{mmol} / \mathrm{L}$ satisfies the above two conditions at the same time. See Table 1 for eluent gradient elution settings.

Table 1. Gradient elution concentration.

\begin{tabular}{cc}
\hline Time $(\min )$ & Concentration $(\mathrm{mmol} / \mathrm{L})$ \\
\hline $0-14$ & 6 \\
$14-25$ & 30 \\
$25-26$ & 6 \\
\hline
\end{tabular}

\subsection{Standard product separation chromatogram}

In this paper, the injection volume is $200 \mu \mathrm{L}$, flow rate is $0.8 \mathrm{ml} / \mathrm{min}$. with the $\mathrm{NaOH}$ concentration in Table 1 for gradient elution and performing measurements, chromatographed results is shown in Figure 1. 


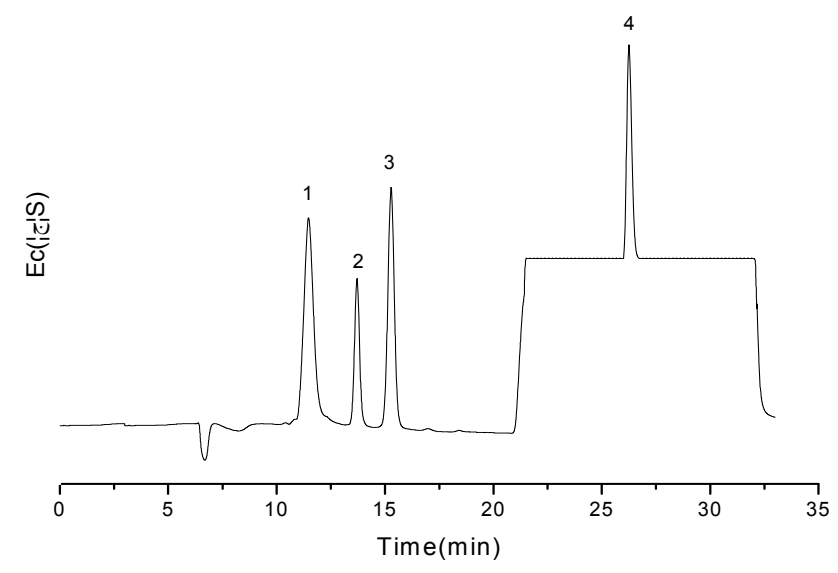

1 Calcium propionate ( $11.44 \mathrm{~min}$ ), 2 Bromate ( $13.71 \mathrm{~min}$ ), 3 Sodium cyclamate ( $15.25 \mathrm{~min}$ ), 4 Acesulfame K ( $26.28 \mathrm{~min}$ )

Fig. 1. Overlaid separation chromatograms of four standards.

\subsection{The method of linear equations, and subject the limit}

Configure the standard curve according to the concentration in Table 2, and obtain the linear equation of the method as seen in Table 3.

Table 2. Standard curve concentration.

\begin{tabular}{ccccccc}
\hline & $\begin{array}{c}\text { Level 1 } \\
(\mu \mathrm{g} / \mathrm{ml})\end{array}$ & $\begin{array}{c}\text { Level 2 } \\
(\mu \mathrm{g} / \mathrm{ml})\end{array}$ & $\begin{array}{c}\text { Level 3 } \\
(\mu \mathrm{g} / \mathrm{ml})\end{array}$ & $\begin{array}{c}\text { Level 4 } \\
(\mu \mathrm{g} / \mathrm{ml})\end{array}$ & $\begin{array}{c}\text { Level 5 } \\
(\mu \mathrm{g} / \mathrm{ml})\end{array}$ & $\begin{array}{c}\text { Level 6 } \\
(\mu \mathrm{g} / \mathrm{ml})\end{array}$ \\
\hline $\begin{array}{c}\text { Calcium } \\
\text { propionate }\end{array}$ & 0 & 1 & 2 & 4 & 10 & 20 \\
$\begin{array}{c}\text { Bromate } \\
\text { Cyclamate }\end{array}$ & 0 & 0.2 & 0.4 & 0.8 & 2 & 4 \\
Acesulfame & 0 & 0.2 & 0.4 & 0.8 & 2 & 4 \\
\hline
\end{tabular}

Table 3. Linear equation of standard curve.

\begin{tabular}{ccc}
\hline & \\
Linear equation ( $\mathrm{Y}:$ peak area $(\mathrm{S} * \mathrm{~min}) \mathrm{X}:$ concentration $(\mu \mathrm{g} / \mathrm{ml}))$ & $\mathrm{R}^{2}$ \\
\hline Calcium propionate & $\mathrm{Y}=0.4034 \mathrm{X}+0.2636$ & 0.9973 \\
Bromate & $\mathrm{Y}=0.7041 \mathrm{X}+0.0124$ & 0.9997 \\
Cyclamate & $\mathrm{Y}=1.0653 \mathrm{X}+0.0133$ & 0.9995 \\
Acesulfame & $\mathrm{Y}=0.4104 \mathrm{X}+0.0722$ & 0.9992 \\
\hline
\end{tabular}

After successive injections of 11 times at the lowest concentration point of the standard curve (level 2) and quantify the peak area, the calculated standard deviations ${ }^{-} \mathrm{X} \pm \mathrm{S}$ of calcium propionate, bromate, average standard sodium cyclamate, acesulfame are respectively $1 \pm 0.021 \mu \mathrm{g} / \mathrm{ml}, 0.2 \pm 0.045 \mu \mathrm{g} / \mathrm{ml}, 0.2 \pm 0.084 \mu \mathrm{g} / \mathrm{ml}, 0.5 \pm 0.052 \mu \mathrm{g} / \mathrm{ml}$; according to the formula $\mathrm{LMD}=3 \mathrm{~S}$, using 3 times the standard deviation divided by the average slope detection limit, one derives the inspection limits are $0.16 \mu \mathrm{g} / \mathrm{ml}, 0.19 \mu \mathrm{g} / \mathrm{ml}$, $0.24 \mu \mathrm{g} / \mathrm{ml}, 0.38 \mu \mathrm{g} / \mathrm{ml}$. 


\subsection{Method accuracy and precision}

Prepare 6 standard solutions of different concentrations of calcium propionate, bromate, sodium cyclamate, and acesulfame K. Inject 6 times and then quantify by the peak area, the standard deviation RSD of the retention times (min) and peak areas ( $\mathrm{S}^{*} \mathrm{~min}$ ) are: calcium propionate $0.13 \sim 0.42 \%$ and $2.14 \sim 4.25 \%$; bromate $0.20 \sim 0.67 \%$ and $1.33 \sim 4.52 \%$; sodium cyclamate $0.14 \sim 0.53 \%$ and $3.57 \sim 5.62 \%$; acesulfame K $0.64 \sim 0.93 \%$ and $0.42 \sim 5.81 \%$.

\subsection{Interfering ion influence}

Because the raw materials of flour products are processed by adding various flavorenhancing substances, sweetener and preservatives, the influence of interfering ions is considered in three aspects. Common anions are arranged in water (containing fluorine chlorine nitrate sulfate ion), flour of common anions (acetate containing hydrogen phosphate citrate Earthroot nitrite ions), flour products sweeteners and preservatives (Contains sodium benzoate, sorbate and saccharin), if the peak sequence cannot be obtained, single needle injection is used to determine the peak time and the sequence of the target peak. The results show that most of the various ions are well separated. Mixing all the interfering ions and one obtains the separated chromatogram in the following figure.

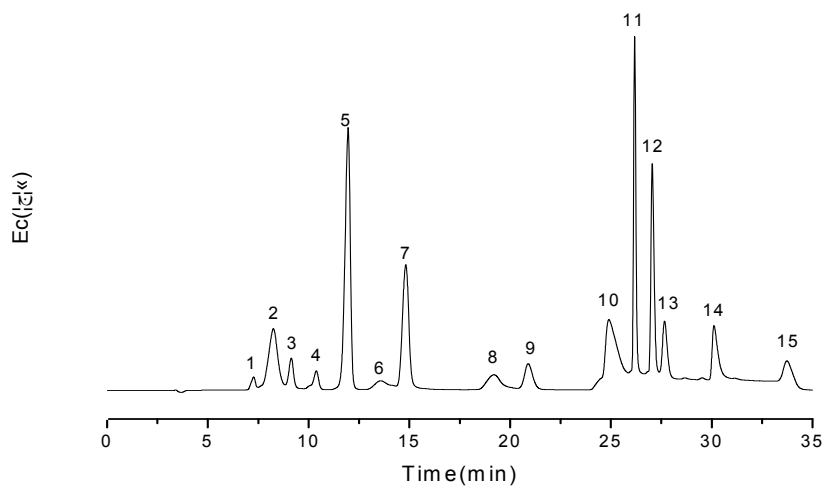

1 Benzoic acid; 2 Formic acid; 3 Acetic acid; 4 Calcium propionate; $5 \mathrm{Cl}$-/ Sodium cyclamate; 6 Bromate; 7 Sorbic acid; $8{ }^{\mathrm{NO}_{2}^{-}} ; 9^{\mathrm{Br}^{-}} ; 10 \mathrm{NO}_{3}^{-} ; 11$ Acesulfame; 12 Sodium saccharin; $13 \mathrm{PO}_{4}^{3-}$; $14 \mathrm{SO}_{4}^{2-} ; 15$ Oxalic acid

Fig. 2. Separation chromatogram of various interfering ions.

It can be seen from the separated chromatogram that only the resolutions of chloride ion and sodium cyclamate are not good, and the peak times of the two overlap. In real sample processing, the interference of chloride ion plasma can be removed by the silver column, whose interference with the targets can then be avoided.

\subsection{Sample recovery rate}

Select a commercially available cake, perform sample pretreatment according to 1.3, determine its background value, and add corresponding concentrations according to the concentration of the four additives to detect the recovery rate, see Table 4. 
Table 4. The recovery rate of four additives.

\begin{tabular}{|c|c|c|c|c|c|}
\hline & $\begin{array}{c}\text { Background value } \\
(\mathrm{mg} / \mathrm{kg})\end{array}$ & $\begin{array}{l}\text { Adding } \\
(\mathrm{mg} / \mathrm{kg})\end{array}$ & amount & $\begin{array}{l}\text { Measured value } \\
(\mathrm{mg} / \mathrm{kg})\end{array}$ & Recovery rate $(\%)$ \\
\hline Calcium propionate & 812.6 & 400 & & 1148.4 & 92.1 \\
\hline Bromate & - & 100 & & 98.7 & 98.7 \\
\hline Cyclamate & 521.5 & 300 & & 830.1 & 102.9 \\
\hline Acesulfame & 123.4 & 100 & & 220.8 & 97.4 \\
\hline
\end{tabular}

\section{4 summary}

An ion chromatography method with simple pretreatment method and large-volume gradient elution injection was established to simultaneously detect four additives in flour products in a single test. Interfering ions were also considered from three aspects to minimize their impact on the target as much as possible, and we tried to ensure that the peak of the target is free of interference. For future perspective, in terms of the compounds that can be dissolved in water, by selecting different eluents, different types of chromatographic columns, and detectors, there is still large potential for inspection of additives by taking advantage of ion chromatography, which is worthy of further exploration.

\section{References}

1. Rules for the Review of 28 Categories of Food Production Licenses of the People's Republic of China (2010 Edition) [Z].

2. National Health and Family Planning Commission of the People's Republic of China. GB 2760-2014 National Food Safety Standard Food Additives Use Standard [S]. Beijing: China Standard Press, 2015.

3. Quality Supervision, Inspection and People's Republic of China and Quarantine. 35382013 SN / T food for export six kinds of synthetic sweeteners method of detecting liquid chromatography - mass spectrometry / mass spectrometry [S]. Beijing: China Standard Press, 2013.

4. National Health and Family People's Republic of China. GB / T 5009. 97 - 20 is 16 Food Safety Standard Determination of sodium cyclohexylamino [S]. Beijing: Chinese Standard Publishing House, 20 is 20

5. National Health and Family Planning Commission of the People's Republic of China. GB 5009.120-2016 National Food Safety Standard Determination of Sodium Propionate and Calcium Propionate in Food [S]. Beijing: China Standards Press, 2020.

6. Determination of bromate in wheat flour. GB/T 20188-2006 General Administration of Quality Supervision, Inspection and Quarantine of the People's Republic of China / National Standardization Administration of China [S], 2006.

7. $\mathrm{Wu}$ Lirong. UPLC method for determination of various pigments, antioxidants, preservatives and sweeteners in food [D]. Jimei University, 2016.

8. Fang, Wang Yan, WANG Yu, Zhou Jun descent, YAN Chao. Solid Phase Extraction High Performance Liquid Chromatography - Evaporative Light Scattering Detection in Food simultaneously. 5 artificial sweeteners in [J]. Chromatography, 2012, 3003 7984. 